\title{
A plicación de técnicas bibliométricas a la gestión bibliotecaria
}

\author{
ELÍAS SANZ CASADO \\ CARMEN MARTÍNMORENO \\ DepartamentodeBiblioteconomíayD ocumentación \\ FacultaddeHumanidades, Co municaciónyD ocumentación \\ UniversidadCarlosiII de Ma drid \\ Tel.(34-1) 62492 42; Fax (34-1) 6249212 \\ E-mail: elias@bib.uc3m.es
}

\begin{abstract}
RESUMEN
Lagestión biblio tecariadebedo tarse, cadavez más, deherramien tasque le permitan conocer las características de sus colecciones y de sus usuarios.Lastécnicasdeanálisisbiblio métrico han desarrollado unacantidad considerabledetalesherramien tasqueayudanaunabuenagestiónalper mitir la medición de distintas características relacionadas con la utilización de los diversos recursos de la biblioteca, así como de sus usuarios. En trelosméto dos deevalua ción queaquíno sólo secitan sino queseexplican yaclaran, sedestacan losin dicado res (seilustralo quesonysedan ejemplos de su uso) tan útiles, por ejem plo, cuan do sequiereco no cerla evolucióndeunaactividadquesehaestado desarrollando enlabiblio teca o sepreten den co no cerlos cam biospro du cidosen los hábi tos dein formación de los usuarios durante un periodo determinado. Se mencionan también los indicadores biblio métricos, quepermiten evaluareluso que seestáhacien do delaco lec ción o co no cerlas carac terísticasdelosusua rioscomo pro duc to resy/ o consumido resdeinformación (sin tenerque con sul tarlos).Y secitan másin dicado res:vidamedia, ín dicedePrice,índice de inmediatez, dispersión, Ley de Bradford, y se analizan otros aspectos: temática de los documentos, tipología de los documentos, etcétera, ydeto dos ellos sein dicael uso queselesha dado ylo que hapodido averiguarse con su aplicación.
\end{abstract}
ABST RACT
Library management must increasingly avail itself of tools which allow the characterization of its collections and users. Bibliometrical analysis techniques have employed a considerable quantity of such tools which help achieve good management by allowing the measurement of different characteristics related to the utilization of distinct library resources, as well as to its users' features. Among the assessment methods which are not only mentioned here but also explained and clarified, are indicators (which are described and examples of their use given) useful, for example, when you want to know the evolution of an activity which has been taking place at the library, or when you mean to know the changes produced in the information habits of users during a given period ot time. Bibliometrical indicators which help to evaluate the

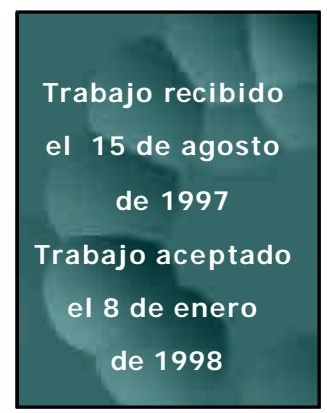


use which is being made of a collection, or to determine the characteristics of users as producers and/ or consumers of information (without having to consult them), are also mentioned. And more indicators are shown: half-life, Price index, immediacy index, dispersion index, Bradford's Law, and other aspects analysed: subject matter of documents, typology of documents, etc. The use which has been made of all these indicators is illustrated, as well as what has been found out with their application.

\section{INTRODUCCIÓN}

H oyen díalamayo ríadelas biblio tecasestásujetaaenormespresio nesrespecto a 1 la gestión desusrecursos deinformación, debido, en mu choscasos, aladismi nución de las inversiones económicas frente al constante aumento del volumen de publicacio nes, denuevosrecursostecno ló gicos, asícomo alasnecesi dadescadavez másso fisticadasdelaco munidad deusuarios. Evidentemente, estaspresionessees tán traduciendo, en muchos centros, en una nueva forma de gestionar los recursos existen tes, con elfin deau men tarsu efi cien cia. En estesen tido, co no cerlasnecesi dadesdeinformación delosusuarios, así como determinarloshábitosen labúsque dayen el uso delain formación quedeman dan, esunatareafun damen tal parapo der desarro llardemodo adecuado gran partedelospro cesosdeevaluación quesereali zan a diario en cual quierbiblio teca. Por ello, tan to el diseño, laplanificación, como la gestión de las bibliotecasdeberán tener en cuen taaquellos criteriosquepermitan realizar correctamente las tareas que se acaban de comentar.

Es conveniente insistir en que la correcta gestión de las bibliotecas pasa de manera obligada por una atención adecuada a sus usuarios, mediante la elaboración de productos y servicios de información útiles para el desarrollo de sus actividades. Para ello, será preciso partir de un buen conocimiento de sus hábitos de información y de sus necesidades específicas, dejando de lado cualquier otro tipo de razones subjetivas que, a veces, responden a deficiencias profesionales que no sólo perjudican al usuario, al no poder satisfacer sus demandas, sino a los propios profesionales de la biblioteca al hacer menos eficientes sus tareas. Por la misma razón, la gestión del centro se deberá de dotar de unas herramientas que le permitan conocer las características de sus usuarios, como: el tipo de información que necesitan, la tipología documental que utilizan, la capacidad idiomática que demuestran, etcétera.

En la gestión bibliotecaria es imprescindible el uso de un gran número de técnicas de análisis, entre ellas las bibliométricas, que complementen las que tradicionalmente usan los bibliotecarios, para conocer la utilización de los recursos de información de sus centros 0 , para determinar aquellas necesidades no satisfechas de sus usuarios. En este sentido, la bibliometría ha desarrollado una cantidad considerable de herramientas que ayudan a la gestión, ya que permite medir distintas características de utilización de los diversos recursos que contiene la bi- 
blioteca, así como de las necesidades que manifiestan los usuarios que acuden a ella. En general, la aplicación de estas técnicas ha proporcionado una enorme ayuda a la hora de definir los sistemas y servicios más adecuados de información de la biblioteca.

\section{MÉTODOS DE EVALUACIÓN}

Hay varias técnicas bibliométricas que se pueden aplicar a la gestión bibliotecaria. En este sentido, quizá sean los indicadores las herramientas de estudio que más se han desarrollado para cumplir este objetivo. Concretamente, fue en la década del 80 cuando se fomentó la investigación sobre la utilización de indicadores para la gestión de este tipo de centros.

A partir de estas herramientas se pueden evaluar muchas de las actividades que se realizan en las bibliotecas, en especial aquellas que están vinculadas con aspectos de tipo cuantitativo. Asimismo, se recomienda la utilización de indicadores cuando se quiere conocer la evolución de las actividades que se han estado desarrollando en la biblioteca o cuando se quieren conocer los cambios que se han producido en los hábitos de información de sus usuarios durante un determinado periodo.

En general, hay que tener en cuenta algunas consideraciones respecto a los indicadores, como: la parcialidad, es decir, cada indicador describe un aspecto concreto del estudio que se está realizando; la convergencia, el uso de un gran número de indicadores permite tener un buen conocimiento de las características de la biblioteca, de los usuarios que está atendiendo, o de los recursos de información que se están utilizando. Estos indicadores son relativos, es decir, se refieren sólo al tipo de biblioteca donde están siendo aplicados o al colectivo de usuarios estudiados, puesto que cada uno de ellos tiene características distintas, y, por tanto, la información que proveen hay que relacionarla exclusivamente con aquellos de su misma tipología.

\section{IN DICADORES BIBLIOMÉTRICOS}

Dentro de los indicadores están aquellos de tipo bibliométrico, que son datos numéricos extraídos de los documentos que publican los investigadores o de los que son solicitados a la biblioteca, y que permiten analizar distintas características de las necesidades y demandas de información existentes dentro de la comunidad de usuarios que la visitan. En este sentido, los indicadores bibliométricos se pueden aplicar, tanto a los recursos documentales de los centros de información, como a los documentos publicados por sus usuarios. En el primer caso, la información obtenida permitirá evaluar el uso que se está haciendo de la colección; mientras que en el segundo, se podrán conocer las características que presentan esos usuarios como productores y consumidores de información. 
Por tanto, para la biblioteca será posible conocer y valorar diferentes aspectos referentes a la utilización que se está haciendo de sus recursos, y de su capacidad para atender las necesidades planteadas por sus usuarios, mediante el uso de los indicadores bibliométricos adecuados. Por su propia definición, éstos se podrán obtener utilizando métodos indirectos (estudio de peticiones de documentos, análisis de citas, análisis de referencias, etcétera), es decir, sin tener que preguntar ni consultar a los usuarios, ya que si se estudian los documentos que publican, que utilizan o los hábitos de información que manifiestan ante diferentes situaciones, se pueden inferir dichas características. Hay que tener en cuenta que aunque los métodos directos (encuestas por correo, entrevistas, etcétera) permiten conocer más a fondo las necesidades reales del usuario, tienen un mayor costo y requieren un mayor tiempo que los indirectos, que se realizan más rápido y ofrecen resultados fiables (Sanz, 1994). En cuanto a los indicadores, hay que señalar que su importancia no estriba sólo en sus valores puntuales, sino en los cambios que se pueden observar en ellos a lo largo del tiempo, pues estas variaciones informarán de las modificaciones que se han ido produciendo en la utilización de los materiales de la biblioteca 0 en la dinámica de los hábitos de información de su comunidad de usuarios.

\section{Indicadores bibliométricos unidimensionales}

Los indicadores bibliométricos unidimensionales estudian una sola característica de los documentos consultados, sin tener en cuenta ningún tipo de vínculo común que pueda existir entre ellos. De estos indicadores destacaremos aquellos que tienen especial relevancia para la gestión bibliotecaria, como son los que se comentan a continuación.

\section{Actualidad de los documentos}

Esta característica se refiere a la tendencia de la colección a caer en desuso, y los indicadores que la valoran, vida media, e índicedePricepermiten conocer la actualidad de los documentos utilizados, y por tanto su obsolescencia. Para una biblioteca es importante conocer el periodo durante el cual los documentos van a ser utilizados y en consecuencia las posibilidades de que sean transformados en nuevo conocimiento. A hora bien, al ser una información directamente relacionada con el tipo de material que utilizan los investigadores de los distintos campos del conocimiento, los bibliotecarios pueden ir retirando aquellos documentos que tengan menor vigencia a zonas menos accesibles.

El envejecimiento que muestra la literatura en cualquier campo del conocimiento se puede determinar mediante el análisis de los años en que se han publicado los documentos utilizados. Esto se calcula por medio de distintos métodos, como por ejemplo: el análisis de las referencias bibliográficas que aparecen en los documentos, el análisis de las citaciones que reciben los do- 
cumentos, y, a partir de la consulta de los documentos que se realiza en una biblioteca.

La obsolescencia de los documentos se puede determinar mediante distintos indicadores. En este sentido, uno de los más utilizados es el de vida media, este término fue establecido por Burton y Kebler (1960), quienes lo definieron como "el tiempo durante el cual fue publicada la mitad de la literatura activa circulante sobre un tema determinado," entendiéndose por literatura activa, aquella que se cita en una bibliografía o es solicitada a una biblioteca.

La obsolescencia se puede medir mediante estudios diacrónicos y sincrónicos. En el primer caso, el análisis indica el tiempo durante el cual podrán ser utilizadas determinadas publicaciones. Para conocer su valor, se selecciona una muestra de documentos publicados en un momento dado, y mediante un análisis de citas, se obtiene la cantidad que han recibido año con año. La vida media así calculada indica el periodo en el que el conjunto de documentos analizados ha recibido la mitad del total de las citas y, según Wallace (1986), este valor permite estimar el tiempo durante el que puede ser utilizada una publicación de un área científica específica.

El estudio sincrónico del envejecimiento de la literatura tiene como objetivo determinar el tiempo que ha pasado desde que se publicó la mitad de la literatura activa más reciente. En este caso, la vida media de los documentos se calcula a partir de su bibliografía, y el valor viene dado por la mediana de los años de antigüedad de los documentos citados en las referencias bibliográficas. La antigüedad así calculada permite conocer los años que han pasado desde que los documentos fueron publicados hasta su utilización.

D esde el punto de vista bibliométrico, es más interesante calcular la tasa de obsolescencia mediante estudios sincrónicos, accediendo a los documentos que han sido publicados. Esta forma de determinar el envejecimiento permite medir la diferente actualidad de los documentos utilizados por los investigadores de los distintos campos científicos, que presenta grandes variaciones de unas áreas a otras, y cuyo conocimiento es fundamental para determinar la política que hay que seguir en los procesos bibliotecarios.

La aplicación de este indicador ha permitido agrupar diferentes disciplinas en función de su envejecimiento más o menos lento. Así, mientras la genética o la física tienen una vida media muy baja, y envejecen rápido (entre 3 y 5 años según los trabajos de Stinson y Lancaster, 1987; Gupta, 1990; Martín y Sanz, 1996), otras disciplinas como la botánica, las matemáticas o la geología la tienen muy alta y envejecen más lentamente, y llegan a tener una vigencia de casi 12 años (Burton y Kebler, 1960). Entre ambos tipos se encuentran las disciplinas de carácter intermedio, como la química, cuyos documentos tienen una vida media de unos 8 años (Burton y Kebler, 1960).

El otro indicador citado utilizado para conocer la obsolescencia de las publicaciones es el índice de Price, que mide el porcentaje de documentos referencia- 
dos en la bibliografía, que tienen 5 años o menos de antigüedad. Para calcularlo, hay que contar el número de documentos contenidos en la bibliografía que tengan 5 años o menos de antigüedad (se considera como año cero el de publicación del documento), y dividirlo entre el número total de referencias. El valor hallado se multiplicará por 100 para dar los resultados en forma porcentual. El resultado informa sobre el mayor o menor porcentaje de documentos recientes que utiliza el usuario para generar nuevo conocimiento.

Se pueden citar múltiples trabajos en los que se ha puesto de manifiesto el interés de este indicador, como los de Moed (1989) en las ciencias de la vida; Wouters y Leydesdorff (1994) estudiaron las diferencias que se dan en distintos campos del conocimiento, ya sea que pertenezcan a las humanidades, ciencias sociales o experimentales, o a las tecnologías. López-Piñero y Terrada (1992a) encontraron que los valores de este indicador para seis revistas biomédicas españolas, oscilaba entre un $20.9 \%$ y un $39.6 \%$, lo que supone una cierta antigüedad en las citas.

\section{Temática de los documentos}

Éste es un aspecto de gran interés para cualquier biblioteca, pues permite conocer la temática de los documentos que son utilizados, así como aquellas otras temáticas que están relacionadas, con el fin de elaborar los productos específicos de información para los distintos colectivos de usuarios que atiende, y dirigir la política de adquisiciones hacia las temáticas más demandadas.

Para estudiar esta característica se utiliza el indicador denominado Temática do amental, y su cálculo se realiza a partir de las referencias bibliográficas de los documentos publicados por los usuarios o demandados en la biblioteca, pues permite analizar la frecuencia de los temas más citados o de los más consultados, y por tanto de aquellos que han sido más utilizados por los usuarios para introducirlos en las distintas actividades que realiza. A partir de ahí, se obtienen los títulos de las revistas citadas, así como la frecuencia con que aparecen. Estos títulos se agrupan por temáticas, para lo cual se puede utilizar la clasificación que hace el Institute for Scientific Information (ISI) con las revistas que recogen sus bases de datos, o bien emplear códigos UNESCO para agruparlas, tal y como hacen varias instituciones españolas.

Entre los trabajos relacionados con este indicador, cabe citar el llevado a cabo por Méndez \& al. (1987) en el que al estudiar las revistas utilizadas por los inmunólogos españoles para dar a conocer sus trabajos, observaron que pertenecían a una gran cantidad de temas diferentes, pero que de alguna manera estaban relacionados con dicha disciplina. Berger y D evine (1990) proponen en su artículo un nuevo método para analizar las colecciones de publicaciones periódicas en el que se combinan distintos criterios de evaluación, entre los que se encuentra la clasificación temática de las mismas. En el estudio que realizaron Gómez \& al. (1990) sobre investigación española en neurociencia, observaron que había un 
amplio espectro de disciplinas relacionadas con el sistema nervioso, dado que numerosos grupos de investigación en esta disciplina publicaban sus resultados en revistas de medicina clínica, fisiología, anatomía, etcétera.

\section{Tipología de los documentos}

Para una biblioteca también es importante conocer el tipo de documentos que consultan y utilizan sus usuarios, pues este dato permite determinar las necesidades de información que tienen, con el propósito de ir elaborando sus perfiles de acuerdo con dichas necesidades. Por todo ello, no hay duda de la importancia de su conocimiento a la hora de elaborar productos de información adecuados a cada tipo de usuario, y por supuesto para definir o redefinir una política de adquisiciones que atienda eficazmente el mayor número posible de demandas.

Mediante el indicador denominado Tipdoǵa Doamental se puede conocer qué tipo de documentos son más utilizados por los usuarios para dar a conocer los resultados de su investigación, o los que consultan para obtener la información que necesitan. El valor de este indicador se determina mediante las frecuencias obtenidas para los distintos tipos de fuentes, a partir de las referencias bibliográficas de los documentos producidos por los investigadores, o de los solicitados a la biblioteca.

Son muchos los trabajos que han estudiado la tipología documental utilizada por los autores de los distintos campos del conocimiento, observándose que el canal formal que mayoritariamente utilizan los de ciencias experimentales para dar a conocer sus contribuciones al conocimiento global, son los artículos en revistas especializadas (Bordóns y Barrigón, 1992; López-Piñero y Terrada, 1992b; Luukkonen, 1992). En un reciente trabajo llevado a cabo sobre la producción científica de los investigadores españoles en genética (Martín y Sanz, 1996) se encontró que dicho medio de trasmisión de conocimientos fue utilizado por los autores en porcentajes superiores al 90\%. Ésta es una característica que diferencia a los científicos de otros campos; así Skelton (1971) señala que las principales fuentes de información de los científicos sociales son las monografías, seguidas de las publicaciones periódicas. Los trabajos llevados a cabo por Cullars (1992) que estudian las características de citación de los humanistas indicaban su preferencia por las monografías frente a otros tipos de publicaciones.

Por todo lo comentado anteriormente, cuanto mayor sea la frecuencia de aparición de las publicaciones periódicas o actas de congresos en sus bibliografías, o en sus consultas a la biblioteca, más efímera será la información que utilicen; por lo que se puede inferir que su campo de trabajo la requiere actualizada y de baja vida media. Mientras que cuando trabajen en temas de mayor obsolescencia, los documentos más citados o demandados serán los correspondientes a una literatura más clásica, como corresponde a la contenida principalmente en las monografías. 
En general, el tipo de documento que se utiliza en las disciplinas de vida media baja es eminentemente efímero, y los artículos de publicaciones periódicas suelen constituir el mayor porcentaje de documentos consultados y utilizados. También suele ser éste el material más utilizado cuando la disciplina tiene una vigencia intermedia, si bien aparece junto a un cierto porcentaje de material clásico. Sin embargo, aquellos campos en que la literatura de consulta presenta un lento envejecimiento, se caracterizan por utilizar preferentemente la monografía como fuente de información.

La biblioteca al conocer este indicador también puede estimar el grado de especialización en las líneas de trabajo de sus usuarios, pues no es lo mismo que citen 0 demanden tesis e informes (característico de usuarios especializados), que congresos, patentes o normas (propio de usuarios que trabajan en ciencias aplicadas y tecnológicas), como han indicado en sus estudios autores como Pérez Álvarez-O ssorio \& al., 1991; Miller, 1992; Luukkonen, 1992.

\section{Visibilidad de los documentos}

Ésta es otra característica que tiene un gran interés para las bibliotecas, dado que permite determinar la importancia de los documentos que poseen, en función de su mayor o menor utilización por los distintos usuarios. De esta forma se puede determinar en cierta medida la calidad de las revistas u otro tipo de publicación, y clasificarlas según su interés científico, es decir, por el impacto que sus trabajos producen en la investigación que se lleva a cabo en su campo específico de conocimiento. Esto permite tener un criterio relativamente objetivo de las mismas y una clasificación que puede utilizarse en el momento de renovar, descartar o suscribirse a nuevos títulos; decisiones de gran importancia para los centros, dado el alto costo que representa la adquisición de estos materiales.

Para conocer la visibilidad de los documentos, se utiliza el indicador Factor de impacto de las revistas, cuyo valor permite conocer la frecuencia con que la información contenida en las publicaciones periódicas es utilizada por los usuarios, para introducirla en su actividad científica y transformarla en nuevo conocimiento. El valor de este indicador se mide a través del número de citas recibidas por la revista que se esté evaluando en un determinado periodo, suponiendo que las más visibles para los usuarios son las que mayor número de citas reciben, y por tanto las que mayor impacto tienen en la comunidad investigadora.

Para calcular el Factor de impacto de una publicación periódica determinada en un año concreto, se divide el número de citas que han recibido los artículos publicados por ella a lo largo de los dos años anteriores, entre el total de artículos publicados en esos dos años.

Esta forma de calcular el indicador provoca una cierta distorsión en la ordenación que se hace de las revistas, pues se benefician aquellas que publican pocos artículos de gran extensión; es decir, las que contienen preferentemente revisio- 
nes, pues aun con cifras similares de citas, al tener menos artículos, el cociente que resulta es mayor, y por tanto muestran mayor Factor de impacto. En este grupo de publicaciones periódicas se pueden incluir las conocidas como Review Progess, Advances etcétera, que pese a no ser publicaciones primarias, por no aportar nuevos conocimientos, suelen ocupar los primeros lugares en las clasificaciones temáticas que se hacen de las revistas, dado que este orden viene determinado por el Factor de impacto de cada una de ellas.

A la hora de interpretar este indicador hay que tener cuidado, pues el Factor de impacto varía de unas disciplinas a otras a causa de las diferencias que existen entre las distintas materias, debido fundamentalmente a los variados hábitos de citación de los científicos de los diferentes campos del conocimiento (Garfield, 1976), por lo que sólo se pueden establecer comparaciones entre las revistas que pertenezcan a las mismas temáticas, es decir, las comparaciones deben ser intradisciplinarias.

En la actualidad, el valor del Factor de impacto sólo se calcula de una 6,000 revistas incluidas en las bases de datos del ISI, y aparece publicado en el Joumal Citation Repats Por este motivo, las bibliotecas especializadas no pueden calcularlo para el resto de su colección, pues aquéllas no son más que una pequeña parte de las más de 75,000 revistas científicas y tecnológicas que se estima que hoy se publican en el mundo.

En los trabajos llevados a cabo que utilizan este indicador, se observan muy variados objetivos. Subramanyan (1975), lo utilizó como criterio de selección de revistas en las bibliotecas. Méndez $\&$ al. (1987) estudiaron, a través de su factor de impacto, la calidad de las revistas de inmunología en las que publicaban investigadores españoles. Baños \& al. (1992) lo utilizaron para valorar las publicaciones biomédicas españolas y su contribución a la ciencia española. Camí đal. (1993) lo emplearon en un estudio llevado a cabo sobre la producción científica española en biomedicina y salud.

El índice de inmediatez es otro indicador de gran interés para las bibliotecas ya que permite conocer el impacto o visibilidad de las publicaciones que utilizan sus usuarios, así como para determinar aquellas que recogen la información más actualizada de un campo científico, y por tanto estimar el mayor o menor interés para las demandas que atienden. El Índice de inmediatez mide el tiempo transcurrido entre la publicación de un documento y su utilización por otros autores en sus trabajos, o lo que es lo mismo, el momento en que es citado.

Como es lógico, cuanto menor sea el tiempo transcurrido entre la publicación de un documento y su citación, o sea su utilización en otro, mayor será el valor del mismo en el sentido de que la comunidad científica lo ha incluido de manera muy rápida en otras líneas de investigación para transformarlo en nuevo conocimiento. 
El Índice de inmediatez de una publicación periódica determinada se calcula dividiendo el total de citas que han recibido los artículos publicados en dicha revista durante el último año, entre el número total de artículos publicados por ella en ese mismo año. El mayor valor de este indicador permite determinar cuáles son las fuentes que recogen la información más actual de una especialidad o disciplina concreta.

El problema que plantea este indicador es que si en un año se publican varios artículos que tienen relación con un mismo tema de investigación, tendrán una mayor probabilidad de ser citados los que se publiquen en los números que salgan a principios de cada año; mientras que aquellos otros que tarden más en ser publicados tendrán menor probabilidad de ser citados durante ese año.

$\mathrm{Al}$ depender de las citas recibidas tampoco el Índice de inmediatez tiene igual valor para todas las disciplinas. Como indicamos líneas amiba, en cada una de ellas la obsolescencia de la información utilizada es distinta. Incluso dentro de una misma disciplina, dependiendo del tipo de investigación que se realice, básica 0 aplicada, también habrá que hablar de diferentes valores para el Índice de inmediatez. Como en el caso del Factor de impacto, los valores anuales de estos índices son publicados anualmente por el Joumal Citation Repats si bien ya hemos indicado que las bases del ISI recogen sólo una pequeña parte de las muchas revistas que se publican. Para evitar este inconveniente, algunas bibliotecas cubanas calculan el Índice de inmediatez de sus documentos teniendo en cuenta el lapso transcurrido desde su ingreso en la colección hasta que son solicitados por los usuarios. Subramanyan (1975) considera que este indicador tiene un gran interés en los procesos de selección de documentos en las bibliotecas.

\section{D ispersión de las publicaciones}

La ley enunciada por Bradford (1934) ha tenido un gran interés para la gestión bibliotecaria, pues permite conocer las revistas más utilizadas por los autores para dar a conocer sus investigaciones, y que son, en la mayoría de los casos, las más demandadas en las bibliotecas. Si se estudian los títulos donde más artículos se publican, se pueden establecer grupos de revistas, con el fin de atender de modo adecuado las necesidades documentales de los usuarios del centro, elaborando productos específicos para ellos, como alertas informativas o difusión selectiva de información.

El indicador de dispersión también permite conocer la frecuencia con la que son consultadas las distintas fuentes documentales. Para calcular su valor hay que hacer un análisis de las referencias bibliográficas, o del número de consultas que han tenido las revistas de la biblioteca, y establecer las frecuencias de los distintos títulos, en función del número de artículos citados o de las demandas realizadas de las revistas. La tabla de frecuencias indica cuántos artículos ha recogido cada uno de los títulos, y aquellas más productivas o más consultadas; las que recojan 
aproximadamente el 50\% de los artículos, formarán lo que se denomina el "núcleo," cuyos títulos deberán pertenecer a la colección.

Otra forma de calcular el núcleo de revistas más productivas es de modo gráfico, representando en una escala semilogarítmica el número acumulado de revistas, frente al número acumulado de artículos recogidos por ellas. Si en la gráfica resultante se traza la recta tangente en el punto de inflexión de la curva, se obtiene el núcleo, el cual estará formado por aquellas revistas que se encuentran situadas a la izquierda del punto de tangencia.

Mediante un estudio de las citas recibidas por una serie de revistas biomédicas, Sengupta (1990) determinó el núcleo de revistas de 8 temáticas, a las que deberían estas suscritas las bibliotecas especializadas en dichas disciplinas. Gómez et al. (1990) encontraron un núcleo de revistas en neurociencia que estaba compuesto por un número pequeño de revistas que eran utilizadas con frecuencia. En el trabajo de Rashid (1991) se propone una pequeña modificación de la ley de Bradford para establecer relaciones entre el número de documentos que publica una revista, y el lugar que ésta podría ocupar en una clasificación temática, teniendo en cuenta su posible interdisciplinariedad.

\section{Barrera idiomática}

En muchas ocasiones se ha indicado que las barreras impuestas por el lenguaje es un problema que tienen los usuarios de ciencias experimentales y tecnológicas; mientras que en ciencias sociales y humanidades, este problema se manifiesta con menor intensidad. Este hecho es relevante, dado que dentro de las ciencias experimentales y tecnológicas, el inglés ha sustituido al francés y al alemán, en lo que se refiere a transferencia de información científica, independientemente de la lengua materna del investigador (Villar, 1988). De tal manera que otros idiomas que antes tuvieron cierta importancia en esta difusión, como son alemán, francés 0 ruso, hoy día prácticamente no se utilizan. De hecho, en la actualidad muchas de las revistas que publican países como Suecia, D inamarca, Japón, etcétera, tienen como idioma de publicación el inglés. No hay que olvidar que a esta situación no están ajenos los productores de bases de datos, preferentemente de habla inglesa, y que abundan los títulos de las revistas que recogen documentos escritos en este idioma (King, 1987). Ante esta situación algunas revistas españolas también han decidido publicar sus artículos en inglés para aumentar su visibilidad y tener más posibilidades de entrar en las bases de datos internacionales (López-Piñero y Terrada, 1992b).

El indicador que permite estudiar las posibles barreras que los científicos tienen ante la información es el conocido como Capacidad idiomática. Para determinarlo se calcula la frecuencia con que los distintos idiomas aparecen en las referencias bibliográficas de las publicaciones, o de los documentos de la biblioteca que han sido consultados. Como es lógico, la aparición de porcentajes muy altos de citas o consultas en el idioma materno, frente a bajos porcentajes en idiomas extranjeros, im- 
plicará una baja capacidad idiomática de los usuarios que redundará, en muchos casos, en el desconocimiento de gran parte de la información científica que se estállevando a cabo fuera del país.

Por todo lo anterior, el conocer los idiomas que pueden entender los usuarios es de vital interés para una biblioteca a la hora de suministrarles la información que demandan, elaborar los productos de información más adecuados para sus necesidades, así como para adecuar la política de adquisiciones de los documentos escritos en los idiomas que les son comprensibles.

\section{Bibliografía nacional utilizada}

Estudiar esta característica permite a una biblioteca conocer en qué medida, la investigación llevada a cabo en un país es utilizada por los usuarios para generar nuevo conocimiento. Por tanto, también permite conocer la permeabilidad que presenta un país a los flujos de información generada fuera de sus fronteras. Su uso es importante en bibliotecas especializadas a la hora de la selección y adquisición de los documentos.

Para conocer qué proporción de bibliografía nacional es utilizada por los científicos, se usa el Índice de aislamiento, indicador cuyo valor viene dado por el porcentaje de bibliografía del propio país que utilizan los científicos, del total de las referencias bibliográficas de sus trabajos. También se puede calcular a partir de los documentos consultados en la biblioteca y que han sido editados por el propio país. Cuanto mayor sea el valor obtenido para este indicador, menor influencia tendrá el conocimiento extranjero en las distintas actividades que se estén realizando en el país. Como es lógico, países con un desarrollo científico medio dependerán en gran medida del conocimiento que se genere en el exterior, por lo que en las bibliografías o en las consultas, aparecerá un gran porcentaje de documentos extranjeros. Sin embargo, aquellos países con un alto nivel de desarrollo no tendrán que recurrir de manera excesiva a fuentes de información externa, si bien en algunos países como Estados Unidos, se han encontrado índices de aislamiento superiores al 50\%, lo que sugiere un cierto desinterés por parte de sus científicos hacia la investigación que otros colegas llevan a cabo en otros países, aspecto que pusieron de manifiesto López-Piñero y Terrada en 1992(c).

A la hora de interpretar el resultado obtenido para este indicador, nuevamente hemos de tener en cuenta que los valores no pueden ser los mismos para todas las disciplinas científicas, por lo que no pueden compararse. Otro hecho que habría de valorarse es la calidad de las revistas, pues si un país no tiene revistas visibles y de buena calidad, los autores tenderán a citar fuentes extranjeras, como sucede en los países del tercer mundo (G ibbs, 1995).

López Piñero y Terrada (1992a) observaron que en 1982, el valor de este indicador presentaba una gran variación entre las revistas médicas españolas nucleares, pues se encontraban títulos como Endorindoǵa o Revista deDiagósticoBidógica que 
presentaban valores mínimos del orden de 4.14\% y 4.95\%, otras como Meeliana Clínica y Revista Clínica Españda mostraban unos valores intermedios, entre el 9 y $10 \%$, y un grupo cuyos valores máximos estaban cerca del 15\%, entre las que citaban Adas Urológicas Españdasy Revista Españda de Enfermedades de A parato Digestiva

\section{Indicadores multidimensionales (mapas)}

Los indicadores multidimensionales permiten tener en cuenta, de modo simultáneo las distintas variables o las múltiples interrelaciones que pueden ser observadas en los documentos, 0 en los hábitos y necesidades de información de los usuarios.

La elaboración de estos indicadores requiere la utilización de técnicas de análisis multivariante. A partir de estas técnicas se pueden elaborar mapas que permiten representar gráficamente diversas características de los usuarios; por ejemplo, los temas en los que están trabajando, las relaciones que mantienen entre ellos 0, las publicaciones periódicas que utilizan para actualizar sus conocimientos o para difundir sus resultados de investigación.

Hay muchos tipos de representaciones gráficas que se pueden obtener a partir del análisis multivariante, probablemente las dos de mayor interés para la gestión bibliotecaria sean el análisis de cluster y el escalado multidimensional. En ambos casos, se pueden representar individuos o variables cuya situación en el mapa dependerá de las similitudes que presenten. Para ello, antes de iniciarse el análisis se deben establecer los criterios que se van a aplicar en el estudio, como son: la selección de las variables que se utilizarán para identificar a los grupos y la selección de la medida de proximidad entre los individuos.

El análisis de cluster tiene por objeto la búsqueda de grupos similares de variables o individuos que se van agrupando en clusters o conglomerados, de tal manera que se pueden crear grupos homogéneos en función de las características observadas. Por su parte, el escalado multidimensional es una técnica que está diseñada para la elaboración de mapas con el propósito de mostrar las relaciones existentes entre individuos o variables en función de las distancias existentes entre ellas. Los mapas obtenidos a partir de esta técnica se pueden representar en una, dos o tres dimensiones, dependiendo de que las variables se sitúen en una línea, en un plano, o mediante puntos en el espacio. Las representaciones obtenidas a partir de un gran número de dimensiones son sumamente complicadas; por este motivo, los dos tipos más utilizados de representación son los de dos y tres dimensiones. De las variables comunes que se analicen con estas técnicas depende que se puedan realizar varios tipos de estudios.

M apas obtenidos a partir del análisis de las citaciones 
En este tipo de mapas se utiliza como vínculo común las citaciones que reciben los autores, los documentos o las publicaciones periódicas, de tal manera que se pueden representar gráficamente redes cognitivas y observar su evolución a lo largo del tiempo.

Los primeros mapas que se realizaron tuvieron como objetivo el estudio de las relaciones establecidas por los autores. Para lo cual, se utilizó como elemento clave de conexión entre ellos, las co-citaciones que recibían los documentos que publicaban, es decir, las citas conjuntas que recibían los autores en publicaciones posteriores (Small, 1976). Este tipo de mapas ha sido analizado en numerosos trabajos, y permite representar gráficamente las líneas de investigación en las que están trabajando los autores. El análisis de las citas conjuntas permite ir agrupando a los autores por temáticas, de tal manera que aquellos que trabajen en campos similares recibirán un elevado número de co-citaciones, y por tanto, se situarán muy cerca unos de otros formando grupos homogéneos.

Algunos trabajos, como los de Small y $\mathrm{G}$ arfield (1986), han dirigido sus objetivos al desarrollo de mapas, con el fin de determinar las relaciones existentes entre las distintas disciplinas científicas, a partir de las co-citaciones como vínculo de unión entre ellas. Para la realización de estos mapas se ha utilizado la información proveniente de los registros de las bases de datos del Institute for Scientific Information (ISI). En este sentido, es conveniente señalar la dificultad que tiene la realización de los mapas de co-citación sin consultar estas bases de datos, puesto que son las únicas que incluyen las referencias bibliográficas en sus registros. Por tanto, este tipo de estudios se ve limitado, en la mayoría de los casos, a aquellos autores que publican sus trabajos en fuentes recogidas por las bases de datos del citado instituto, el que presenta una fuerte inclinación hacia las publicaciones de países anglosajones.

Del mismo modo que con los autores o con los documentos, se pueden realizar mapas con las publicaciones periódicas, con lo cual se puede identificar y evaluar el núcleo de revistas científicas dedicadas a una determinada disciplina, o de las más consultadas por los usuarios de una biblioteca. En las representaciones de este tipo de mapas, la distancia entre las publicaciones periódicas dependerá del número de citas conjuntas que reciban o del número de veces que hayan sido consultadas conjuntamente en una biblioteca; de tal manera que aquellas que tengan una frecuencia similar se situarán cerca unas de otras, formando grupos. McCain (1991) ha contribuido de manera importante al desarrollo de mapas a partir de las co-citaciones que recibían las publicaciones periódicas. Sus trabajos han permitido definir y representar gráficamente el núcleo de revistas de mayor interés en determinadas disciplinas científicas.

\section{M apas obtenidos a partir del análisis de las co-palabras}

O tros estudios que han sido analizados recientemente, por el gran interés que tienen para la elaboración de mapas temáticos, son los realizados a partir de co- 
palabras, es decir, a partir de la aparición conjunta en los documentos de los mismos descriptores, palabras clave, términos de los resúmenes 0, incluso, determinadas palabras del título de los trabajos publicados (Callon đal., 1995).

Es conveniente señalar que, aunque este tipo de mapas se puede realizar a partir de las características de los documentos que se acaban de comentar, los mapas realizados a través de descriptores han mostrado tener una mayor precisión para representar los temas de los que tratan los documentos, puesto que a diferencia de las palabras clave, que son asignadas por los autores, y, por tanto, la misma palabra puede no describir siempre los mismos contenidos; los descriptores tienen una asignación más objetiva, ya que provienen de un vocabulario controlado, y, por ello, cada descriptor siempre estará asociado a los mismos conceptos.

En estos mapas, como en el caso anterior, los descriptores se situarán cerca unos de otros en función del número de veces que aparezcan juntos en los documentos. En este sentido, el número de co-ocurrencias de los descriptores, palabras clave, etcétera, en los trabajos científicos permitirá que se formen grupos temáticos que se van a ir situando en distintos niveles de agregación, dependiendo del grado de similitud que presenten.

Evidentemente, la realización de este tipo de mapas representa una enorme ventaja respecto a los de co-citación, puesto que su realización no estará limitada por la utilización de las bases de datos del Institute for Scientific Information (ISI), sino que se puede utilizar un gran número de bases de datos específicas o multidisciplinarias.

\section{BIBLIOG RAFÍA}

BAÑO S, J. E.; I. Casanovas; E. Guardiola; F. Bosch. “Análisis de las revistas biomédicas españolas mediante el fac tordeim pacto." Medioma dínica(Barc), 1992, 99: 96-99.

Berger, M.; J. D evine. "Serials evaluation: An innovative approach." Speial Libranies 1990 Summer: 183-188.

BORD ÓNS,M.;S.Barrigón. "Biblio metricanalysisofpublications os Spanish pharmacologists in the SCI (1984-89). Part. II. " Säenammics 25 ( ): 425-446.

BRADFORD , S.C. "Sources of information on specific subjects". Engineting 1934, vol. 137: 85-86.

Burto N, R.; R. W. Kebler. "The "half-life" of some scientific and technical literatures." Anmican Doumentation, 1960, 11: 18-22. 
CALlon, M.; J.P. Courtial; H. Penan. Cieniomenáa Gijón, ediciones TREA, 1995.

CAMÍ,J.;M.T.Fernández;Y.Gómez. “Lapro duc ción científicaespañolaenbio medici naysalud. Un estu dio atravésdel Scien ceCitation In dex (1986-1989)." MedidnaClíni ca(Barc), 1993, 101 (19): 721-731.

Cullars, J. "Citation characteristics of monographs in the fine arts." LibraryQuartery, 1992, 62(3): 325-342.

GARFIELD , E. “Significant journals of science." Nature, 1976, 264: 609-615.

GiBBS, W.W. “Ciencia del tercer mundo." InvestigacónyCienaia 1995, 231:70-79.

Gó MEZ, I.; E. Sanz; A. Méndez. "Utility of bibliometric analysis for research policy: a case study of Spanish research in neuroscience," Reerch Pdigy, 1990, 19: 457-466.

GuPTA, U. "Obsolescence of physics literature: exponential decrease of the density of citationsto PhusicalReviewarticles with age," Jarnal oftheAmericanSodidyofInformation Säere, 1990, 41 (4): 282-287.

KING ,J. “A review of biblio metric and otherscien cein dicatorsand theirrolein research evaluation." Jamal of Information Säence, 1987, 13: 261-276.

LÓ PEZ PiÑeRo J.M.;M.L.Terrada. “Losin dicado resbiblio métricosylaevaluación de la actividad médico-científica.(IV). La aplicación de los indicadores." Mediảa Clírica (Barc), 1992 (a), 98: 384-388.

- - -. "Losindicado resbiblio métricosylaevaluación delaactividadmédico-cientifica. (II).Laco municación cien tíficaen las distintasáreasdelascien ciasmédicas." Medidna Clínica(Barc), 1992 (b), 98: 101-106.

- - -. "Losindicado resbiblio métricosylaevaluación delaactividadmédico-científica. (III).Losin dicado resdepro ducción, circulaciónydispersión, consumo delainforma ción y repercusión." Međiaina Clínica (Barc), 1992 (c), 98: 142-148.

LUUK KONEN, T. "Is scientists' publishing behaviour reward seeking? " Säentonetrics, 1992, 24( ) 297-319.

MarTínMo RenO,C.;E.SanzCasado. "Pro duccióncien tíficaespañolaeneláreadege nética," Reissta Espeñda deDoumentacoón Científica, 1996, 19 (4): 377-391.

McCAIN,K.W. “Corejournalnetworksand co citation maps: Newbiblio metrictoolsfor serials research and management." LibraryQuartery, 1991, 61(3): 311-336.

MÉND EZ,A.;I.Gó mez;E.Sanz;E. Mo rales. “Lain mu no lo gíaespaño laatravés desus publicaciones." Inmmoloǵa 1987, 6(3): 122-133.

MILLER, R. "Thein fluen ce of primarytask on R\&D labo ratoryevaluation:acom parative bibliometric analysis." R\&D Managment, 1992, 22: 3-19.

MoED,H.F. "Biblio metricmeasurementof research performan ceand Price'stheoryof difference among sciences." Säeniomerics 1989, 15 (1): 473-483. 
PÉ Rez Álvarez-O sso Rio, J.R.; M. Y. Gómez Caridad; M. J. Martin Sempere; C. Galban Ferrus; M. C. Urdín Caminos; A. Y. So brado Presa. "Lapro ducción delaUniversidad española en física, reflejada en las publicaciones españolas y extranje ras." Reista españda de Doumentación Científica, 1991, 14: 428444.

RADISH, F.F. "Bibliometric analysis as a tool in journal evaluation." SeialsLibrairan, 1991, 20 (2/ 3): 55-64.

SANZ CASAD o, E. Manual de estudios deusuarios 1994, Madrid, Fundación Germán Sánchez Ruipérez.

SENG UPTA, Y.N. "Biblio metricsandiden tification of coreperiodicals." Heald of Library Saiene, 1990, 29 (3/ 4): 226-247.

SKELTO N, B. Companison of results of siencestudes with investigation into infomation requirements of the social sience Bath University, Bath University Library, 1971.

SMALL, H.G.; E. Garfield. "The geography of science: discipli nary and natio nal mappings." JournalofInformationSaience, 1986, 11: 147-159.

STINSO N, E.R.; F. W. Lancaster. "Synchronous versus diachronousmethodsin measurementof obso lescen cebycitationstu dies," Jaumal of Infomation Saience, 1987, 13: 136-145.

SUBRAMANYAN, K. “Criteriaforjournal selection." SpedalLibra ries 1975, 66 (8): 367-371.

V ILLAR,J. “Elinglés,idio main ternacionalenmedicina.” MedClin (Barc), 1988, 91: 23-24.

WALLACE, D. "The relationship between journal productivity and obsolescence." Jamal of theAmerican Socity for Infomation Saience, 1986, 37(3): 136-145.

Wo uters, P.; L. Leydesdorff. "Has Price's dream come true: Is sciento metricsahard scien ce?" Säentomaris 1994, 31 (2): 193222.

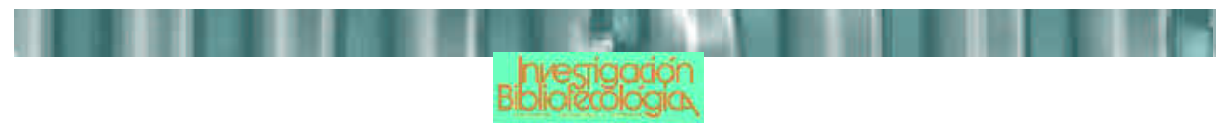

